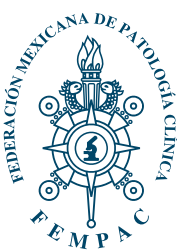

Palabras clave: COVID-19,

drepanocitosis, células falciformes, inducción a drepanocitos.

Keywords: COVID-19, sickle cell, sickle cell, sickle cell induction.

* Laboratorio Clínico Velázquez, Berriozábal, Chiapas, México, Instituto de Salud del

Estado de Chiapas, Centro Estatal de la

Transfusión Sanguínea, Tuxtla Gutiérrez, Chiapas México. ${ }^{\ddagger}$ Operatividad, análisis de muestras, revisión de manuscrito; Laboratorio Clínico Velázquez, Berriozábal, Chiapas, México.

$\S$ Universidad Autónoma de Chiapas, Escuela de Ciencias Químicas Sede Ocozocoautla de Espinosa, Chiapas, México.

Correspondencia: Dr. en C. Martín Velázquez-Gómez 10 a Sur Poniente Núm. 254, Barrio Candelaria, Berriozábal, Chiapas, México, 29130, Tel: 9611661137 E-mail: velazquezgm@gmail. com

Recibido: 12/09/2020 Aceptado: 01/10/2020

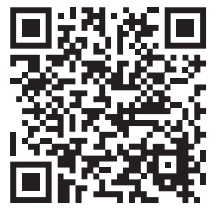

\title{
COVID-19 y drepanocitosis
}

\author{
COVID-19 and sickle cell disease
}

\author{
Velázquez-Gómez Martín, * Ruiz-Pérez Jaqueline, ${ }^{\ddagger}$ Culebro-Cruz Erika Patricia§
}

\section{RESUMEN}

Introducción: COVID-19 es una enfermedad respiratoria aguda caracterizada por fiebre, tos seca, cansancio, congestión nasal, cefalea, anosmia e incluso dificultad para respirar. La drepanocitosis es un padecimiento hereditario por la presencia de hemoglobina $\mathrm{S}(\mathrm{HbS})$, en el que cuando el oxígeno es bajo, los glóbulos rojos se deforman, lo que ocasiona oclusión de la microcirculación y genera hiperreactividad de vías aéreas superiores e hipertensión pulmonar. Objetivo: Conocer la importancia de la inducción a drepanocitos en pacientes sospechosos de COVID-19 u otras enfermedades respiratorias. Material y métodos: Se analizaron casos sospechosos compatibles con COVID-19 de abril-agosto 2020, mediante biometría hemática, inducción a drepanocitos, frotis sanguíneo, electroforesis de hemoglobina, glucosa, colesterol, triglicéridos, proteína $\mathrm{C}$ reactiva, reacciones febriles y antidengue Duo. Resultados: De 309 pacientes, 225 (72.8\%) fueron hombres y 84 (27.2\%) mujeres; la edad promedio fue de $53.2 \pm 8$ años de edad, se detectaron seis (1.94\%) casos de drepanocitosis (cinco heterocigotos y un homocigoto) con índices hematológicos normales, confirmados por electroforesis de hemoglobina. Conclusiones: La inducción a drepanocitos y lectura de frotis sanguíneo se debe realizar a todos los pacientes con o sin anemia, en especial aquéllos donde los niveles de oxígeno en sangre estén comprometidos.

\begin{abstract}
Introduction: COVID-19 is an acute respiratory illness characterized by fever, dry cough, tiredness, nasal congestion, headache, anosmia, and even shortness of breath. Sickle cell disease is a hereditary condition due to the presence of hemoglobin $S(\mathrm{HbS})$ which, when oxygen is low, the red blood cells are deformed causing occlusion of the microcirculation generating hyperstimulation of the upper airways and pulmonary hypertension. Objective: To know the importance of sickle cell induction in patients suspected of COVID-19 or other respiratory diseases. Material and methods: Suspicious cases compatible with COVID-19 were analyzed from April-August 2020, by hematic biometry, sickle cell induction, and blood smear, electrophoresis of hemoglobin, glucose, cholesterol, triglycerides, C-reactive protein, febrile reactions and anti-dengue Duo. Results: Of 309 patients, $225(72.8 \%)$ were men and $84(27.2 \%)$ were women, the mean age was $53.2 \pm 8$ years of age, $6(1.94 \%)$ cases of sickle cell disease were detected ( 5 heterozygous and 1 homozygous) with indices normal hematological tests, confirmed by hemoglobin electrophoresis. Conclusions: Sickle cell induction and blood smear reading should be performed in all patients with or without anemia, especially those where blood oxygen levels are compromised.
\end{abstract}

\section{INTRODUCCIÓN}

T os coronavirus (CoV) son una gran familia $\_$de virus que causan enfermedades que van desde el resfriado común hasta enfermedades más graves. COVID-19 es una enfermedad causada por el coronavirus SARS-CoV-2, que fue notificado por primera vez en Wuhan, China, el 31 de diciembre de 2019. La epidemia de COVID-19 fue declarada por la OMS como una emergencia de salud pública de preocupación internacional el 30 de enero de 2020; en la actualidad, es una pandemia que afecta a muchos países de todo el mundo. ${ }^{1}$

Los síntomas más habituales son fiebre, tos seca y cansancio; otros menos frecuentes son los dolores y molestias, congestión nasal, dolor de cabeza, conjuntivitis, dolor de garganta, diarrea, pérdida del gusto o el olfato y erupciones cutáneas o cambios de color en los dedos de las manos o los pies. Estos síntomas suelen ser leves y comienzan gradualmente. Algunas de las personas infectadas sólo presentan síntomas leves. La mayoría (alrededor de 80\%) se recuperan de

Citar como: Velázquez-Gómez M, Ruiz-Pérez J, Culebro-Cruz EP. COVID-19 y drepanocitosis. Rev Mex Patol Clin Med Lab. 2020; 67 (3): 124-128. https://dx.doi.org/10.35366/96675 
la enfermedad sin necesidad de tratamiento hospitalario, solamente una de cada cinco personas que contraen COVID-19 terminan presentando un cuadro grave y experimenta dificultades para respirar. Las personas mayores y las que padecen afecciones médicas previas como hipertensión arterial, problemas cardiacos o pulmonares, diabetes o cáncer tienen más probabilidades de presentar cuadros graves. Sin embargo, cualquier persona puede contraerlo y debutar gravemente enferma. ${ }^{2}$

El término drepanocitosis se utiliza para designar a un grupo de anemias hemolíticas congénitas con hemoglobina $\mathrm{S}(\mathrm{HbS}) .{ }^{3} \mathrm{La} \mathrm{HbS}$ se caracteriza por la formación de células drepanocíticas, la anomalía estructural se encuentra en la cadena de globina $\beta$ en la posición 6 con una sustitución de aminoácidos: ácido glutámico por valina. La sustitución de un aminoácido genera cambios en la solubilidad molecular de la hemoglobina. ${ }^{4}$

Las complicaciones clínicas generalmente se manifiestan en estado homocigoto siendo las principales: crisis vasooclusiva, crisis hemolítica, alteraciones óseas, entre otras. La condición heterocigota en ocasiones pasa desapercibida y es diagnosticada como hallazgo. ${ }^{5} \mathrm{La} \mathrm{HbS}$ polimeriza en condiciones de baja tensión de oxígeno y deforma al hematíe, esto provoca acortamiento de la supervivencia y oclusión de la microcirculación, lo que da lugar a la anemia hemolítica crónica y a las crisis vasooclusivas dolorosas (CVD), que marcan en gran medida el cuadro clínico de la enfermedad, lo que puede inducir la aparición de hematuria e infartos esplénicos en estos heterocigotos. ${ }^{6}$ Estos procesos incluyen disfunción del endotelio vascular, deficiencia funcional del óxido nítrico (NO), inflamación, estrés oxidativo, daño en la reperfusión, hipercoagulabilidad, aumento de la adhesión al endotelio de los neutrófilos y activación de las plaquetas. ${ }^{7}$

La patología respiratoria aguda y crónica aparece frecuentemente en pacientes con drepanocitosis y constituye un importante factor de riesgo para una muerte prematura. Entre éstas se encuentran el síndrome torácico agudo (STA), la hiperreactividad de vías aéreas superiores y la hipertensión pulmonar (HTP), en los pacientes con drepanocitosis se han descrito alteraciones de la función pulmonar. ${ }^{8-10}$

Se estima que la prevalencia de portadores de células falciformes en 25 estados europeos es de aproximadamente $1 / 150$. En África central y occidental 15-25\%, en las Indias occidentales francesas $10-15 \%$ y en zonas mediterráneas $1-15 \%$, se ha observado una mayor prevalencia en zonas que están o han estado afectadas por

\begin{tabular}{|c|c|c|c|c|c|c|c|}
\hline \multirow[b]{2}{*}{ Parámetro } & Caso 1 & \multicolumn{2}{|c|}{$\begin{array}{c}\text { Caso } 2 \\
\text { Hombre (años) }\end{array}$} & Caso 4 & \multicolumn{2}{|c|}{$\begin{array}{c}\text { Caso } 5 \\
\text { Mujer (años) }\end{array}$} & \multirow{2}{*}{$\begin{array}{c}\text { Valores de } \\
\text { decisión clínica * }\end{array}$} \\
\hline & 37 & 28 & 42 & 77 & 32 & 69 & \\
\hline Hemoglobina (g/dL) & 13.7 & 14.1 & 15.9 & 6.3 & 13.1 & 10.1 & $12.7-16.6$ \\
\hline Eritrocitos $(x 106 / \mu \mathrm{L})$ & 4.75 & 4.35 & 5.33 & 1.86 & 4.73 & 3.34 & $4.2-5.5$ \\
\hline Leucocitos $(/ \mu \mathrm{L})$ & 10,900 & 11,100 & 12,200 & 1,400 & 10,800 & 11,000 & $4,500-11,000$ \\
\hline $\begin{array}{l}\text { Neutrófilos segmen- } \\
\text { tados }(/ \mu \mathrm{L})\end{array}$ & 6,800 & 7,100 & 7,950 & 476 & 7,664 & 9,393 & $1,800-7,000$ \\
\hline Linfocitos $(/ \mu \mathrm{L})$ & 1,100 & 959 & 837 & 812 & 897 & 606 & $1,000-4,800$ \\
\hline Plaquetas $(/ \mu \mathrm{L})$ & 179 & 112 & 148 & 16 & 228 & 136 & $150-450$ \\
\hline Glucosa (mg/dL) & 151 & 94 & 81 & 138 & 133 & 535 & $70-110$ \\
\hline Colesterol (mg/dL) & 362 & 139 & 212 & 165 & 130 & 312 & $<200$ \\
\hline Triglicléridos (mg/dL) & 566 & 472 & 138 & 89 & 95 & 299 & $<150$ \\
\hline $\mathrm{PCR}(\mathrm{mg} / \mathrm{L})$ & 48 & 24 & 12 & 96 & 96 & 192 & $<6$ \\
\hline Reacciones febriles & $\begin{array}{l}\text { Positivo } \\
(1: 160)\end{array}$ & Negativo & Negativo & Negativo & $\begin{array}{l}\text { Positivo } \\
(1.80)\end{array}$ & Negativo & $<1.80$ \\
\hline $\begin{array}{l}\text { Anti-Dengue NS1, } \\
\text { lgM e IgG }\end{array}$ & Negativo & Negativo & Negativo & Negativo & Negativo & Negativo & Negativo \\
\hline
\end{tabular}


Figura 1:

Inducción a drepanocitos con metabisulfito de sodio $\left(\mathrm{Na}_{2} \mathrm{~S}_{2} \mathrm{O}_{3}\right)$ al $2 \%$, observados a los 30 y 60 minutos con el objetivo 10 y $40 x$ (microscopio óptico Velab VE-B2).
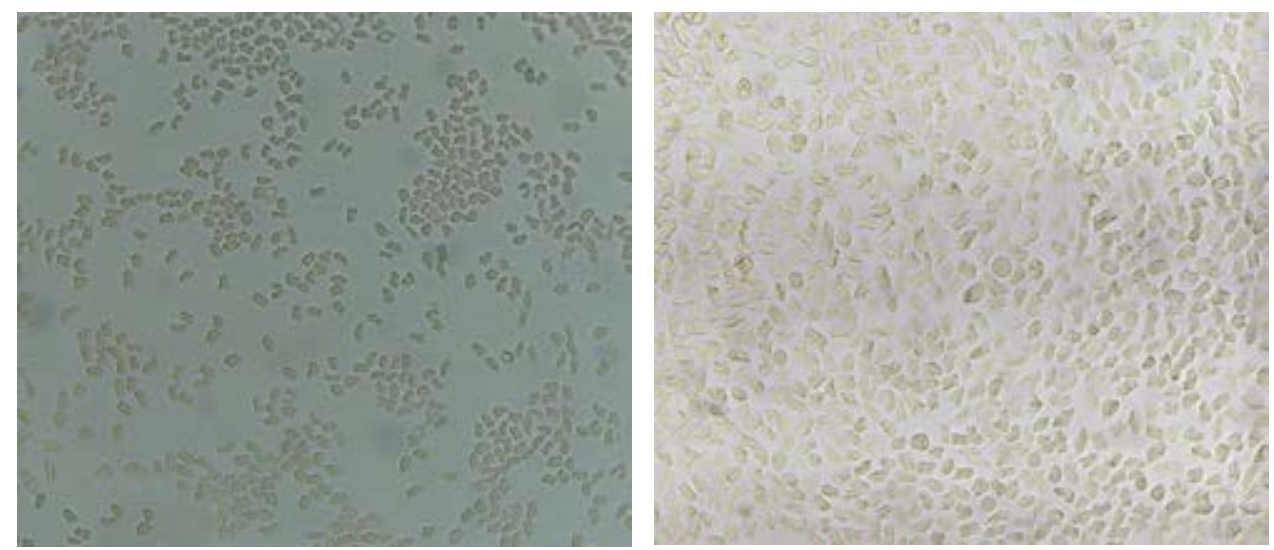

malaria, porque el rasgo ofrece protección contra malaria perniciosa. ${ }^{11}$

El objetivo del presente trabajo es dar a conocer la importancia de realizar inducción a drepanocitos a pacientes con o sin anemia, especialmente a casos sospechosos con síntomas clínicos de COVID-19 u otras enfermedades respiratorias.

\section{MATERIAL Y MÉTODOS}

Se trata de un estudio descriptivo de casos de pacientes que, por indicaciones médicas, acudieron a la realización de biometría hemática completa $(\mathrm{BHC})$, proteína $\mathrm{C}$ reactiva (PCR), glucosa, colesterol, triglicéridos, tiempos de coagulación, reacciones febriles y en algunos de ellos la prueba antidengue NS1-IgM-IgG como seguimiento y/o diagnóstico diferencial de COVID-19, al laboratorio de análisis clínicos «Velázquez», del Municipio de Berriozábal, Chiapas, México, en el periodo de abrilagosto del 2020.

Como procedimiento interno de dicho laboratorio, se lleva a cabo la realización de inducción a drepanocitos a todos los pacientes con BHC con o sin anemia. Se depositaron $10 \mu \mathrm{L}$ de metabisulfito de sodio $\left(\mathrm{Na}_{2} \mathrm{~S}_{2} \mathrm{O}_{3}\right)$ al $2 \%$ en un portaobjetos, se agregaron $5 \mu \mathrm{L}$ de sangre fresca con EDTA, se mezcló, se colocó un cubreobjetos de 22 $\times 22 \mathrm{~mm}$, se sellaron los bordes con resina, se dejaron reposar por dos horas y cada 30 minutos se observaron al microscopio con el objetivo 40x.

Los frotis sanguíneos fueron teñidos con colorante de Wright, después se realizó un montaje con resina sintética con cubreobjetos de $22 \times 50 \mathrm{~mm}$ y se observaron con el objetivo de inmersión 100x.

Las muestras con inducción a drepanocitos positiva se enviaron a un laboratorio de referencia para la realización de electroforesis de hemoglobina a pH alcalino en membrana de acetato de celulosa en una cámara electroforética a $200 \mathrm{~V}$ (voltaje constante) y otra a $\mathrm{pH}$ ácido con soporte de agar en citrato.

Los datos fueron analizados con medidas de tendencia central y dispersión con el programa estadístico SPSS versión 19.

Consideraciones bioéticas: todos los procedimientos fueron adecuados a las recomendaciones para la investigación biomédica de Helsinki de la Asociación Médica Mundial ${ }^{12}$ y a la Ley General de Salud de México, ${ }^{13}$ este estudio es de riesgo mínimo, como lo estipula la mencionada ley. Para proteger los datos de los pacientes, no se incluyó información personal de ninguno de ellos.

\section{RESULTADOS}

De un total de 309 pacientes referidos por un médico, $225(72.8 \%)$ fueron hombres y $84(27.2 \%)$ mujeres, la edad promedio fue de $53.2 \pm 8$ años de edad, todos originarios de Berriozábal, Chiapas. Se presentaron con síntomas sospechosos de COVID-19, fiebre de 37.5 a 38 ${ }^{\circ} \mathrm{C}$, cefalea moderada a severa, artralgias, dolor de garganta, tos seca (algunos con flemas) y pérdida del olfato (anosmia) de cinco a siete días de evolución.

Se identificaron seis (1.94\%) casos de drepanocitosis (cinco heterocigotos y un homocigoto), tres hombres y tres mujeres con una edad media de 47.5 años. La mayoría de los pacientes presentaron hemoglobina e índices hematológicos (VCM, HCM, CMHC) normales, leve leucocitosis a expensas de neutrofilia absoluta y linfopenia, niveles de plaquetas normales, PCR aumentado, hiperglucemia y dislipidemia, tiempos de coagulación normal, reacciones febriles con leve alteración en los títulos para tífico O y Proteus OX19 (Tabla 1).

La formación de células en forma de media luna con metabisulfito de sodio se presentó al cabo de 30 a 60 
minutos y en el frotis sanguíneo se observaron eritrocitos con hemoglobina polarizada (drepanocitos), así como escasos poiquilocitos y esferocitos (Figuras 1 y 2).

La electroforesis de hemoglobina demostró la presencia de $\mathrm{HbS}$ en una concentración de $37.5 \pm 1.2 \%$ para los pacientes heterocigotos, mientras que para el paciente homocigoto (caso 4) fue de $73.1 \%$ de HbS (Tabla 2).

\section{DISCUSIÓN Y CONCLUSIÓN}

A nivel mundial, las hemoglobinopatías representan $71 \%$ de los problemas sanitarios en 229 países considerados por la OMS, donde cada año nacen 330,000 niños afectados, de los cuales $83 \%$ corresponden a las anemias de células falciformes (drepanocítica) y $13 \%$ al caso de las talasemias, lo que provoca $3.4 \%$ de muertes en niños menores de cinco años. ${ }^{14}$ En México, algunas publicaciones indican una prevalencia entre 1 y $2 \%$ de portadores de $\mathrm{HbS}$, especialmente en regiones del norte de Veracruz y Puebla, ${ }^{15}$ similar a lo que en este estudio se observa (1.94\%).

La drepanocitosis forma parte de las enfermedades $\operatorname{raras}^{11}$ y se clasifica dentro de las anemias normocíticas normocrómicas, ${ }^{16}$ pero los individuos asintomáticos (heterocigotos) presentan una $\mathrm{BHC}$ sin anemia y sin alteraciones en los índices hematológicos (VCM, HCM, $\mathrm{CMHC}$ ) y en la morfología de sangre periférica, a excepción de los homocigotos. En este trabajo se demuestra esa normalidad en los pacientes portadores asintomáticos, pues se identificaron de manera fortuita, debido a que en dicho laboratorio se tiene como protocolo interno realizar inducción a drepanocitos a todos los pacientes que se les solicita $\mathrm{BHC}$, resulten o no con anemia.

Unos de los escenarios de gravedad de COVID-19 son la diabetes e hipertensión; así como la presencia de neutrofilia y linfopenia, ${ }^{2}$ datos de laboratorio que se presentaron en la mayoría de los casos sospechosos. De igual manera, el aumento de la PCR (mayor $>15 \mathrm{mg} / \mathrm{L}$ ) estuvo presente en los casos, la ausencia de títulos altos en las reacciones febriles y la no reactividad al antígeno NS1 para Dengue descartaron una probable infección aguda de tipo bacteriana o viral para DENV.

Tanto en la literatura científica como en las normas oficiales mexicanas, no existe la imposición de hacer el tamizaje para esta enfermedad como algo rutinario, el diagnóstico es complicado, porque no hemos logrado establecer la inducción de drepanocitos como una prueba que se realice simultáneamente a la $\mathrm{BHC} ;{ }^{16}$ sin embargo, en este estudio se puede observar que la presencia de esta enfermedad puede llegar a ser tan común y diagnosticar principalmente las formas no graves (heterocigotas), considerando que son pacientes asintomáticos y con una BHC totalmente normal.

El diagnóstico de los heterocigotos es complejo, ya que la morfología observada en los frotis sanguíneos se limita a escasos codocitos, ocasionales eritrocitos con hemoglobina polarizada y raramente se logran observar los drepanocitos; ${ }^{16}$ pero en los casos identificados en este análisis durante cinco meses, todos los heterocigotos presentaron drepanocitos, lo cual sugiere que la baja en la tensión de oxígeno por COVID-19 u otra enfermedad respiratoria que conlleva a neumonía, posea una influencia directa y empeore la enfermedad, lo que puede llegar a ser fatal en los pacientes. Cabe señalar que la paciente identificada como homocigota falleció a las pocas horas de haber sido hospitalizada.

La electroforesis de hemoglobina y el estudio familiar de los casos identificados por inducción a drepanocitos, son las únicas formas para confirmar el diagnóstico de la presencia de la $\mathrm{HbS}$, donde la concentración para los heterocigotos oscila entre 35 y $50 \%$ y los homocigotos $>50 \%{ }^{16}$ Cinco casos identificados en este estudio presentaron una concentración de HbS $37.5 \pm 1.2 \%$ y uno de ellos $73.1 \%$.

Los pacientes con síndrome respiratorio agudo, donde la tensión de oxígeno se encuentra comprome-
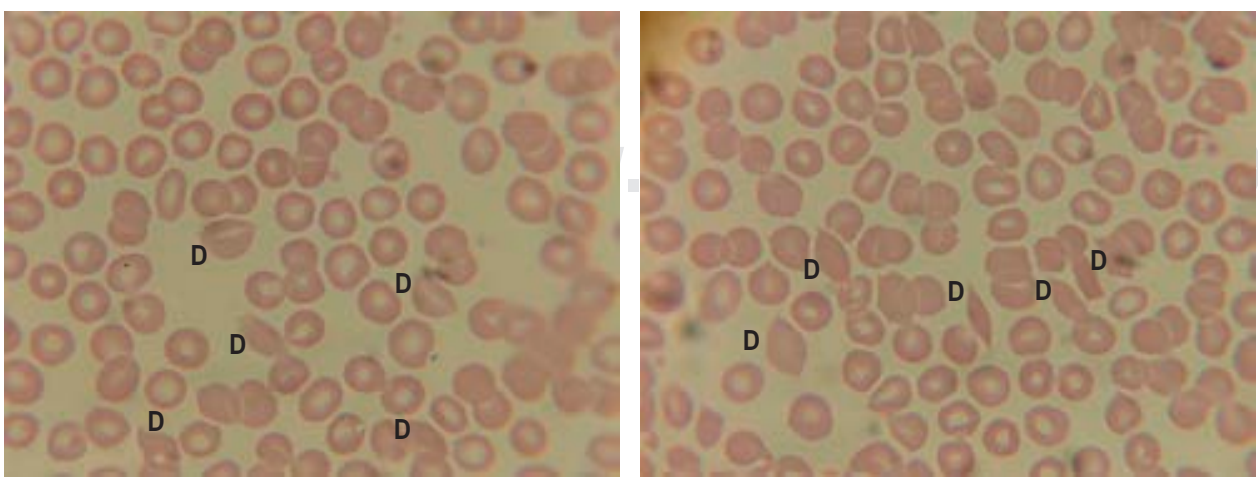

Figura 2:

Frotis de sangre periférica con drepanocitos (D) de paciente homocigota y heterocigota, teñida con colorante de Wright marca Ojo Biónico y observada con el objetivo 100x. 
Tabla 2: Concentraciones de los diferentes tipos de $\mathrm{Hb}$ en los casos de drepanocitosis con síntomas clínicos de COVID-19, Berriozábal, Chiapas, México, 2020.

\begin{tabular}{ccccc} 
& \multicolumn{4}{c}{ Tipo de hemoglobina $(\mathrm{Hb}) \%$} \\
\cline { 2 - 5 } Casos & $\mathrm{HbA}$ & $\mathrm{HbA} 2$ & $\mathrm{HbF}$ & $\mathrm{HbS}$ \\
\hline 1 & 59.8 & 1.6 & 0.5 & 38.1 \\
2 & 60.9 & 1.5 & 0.0 & 37.6 \\
3 & 61.7 & 1.8 & 0.1 & 36.4 \\
4 & 23.45 & 2.9 & 0.55 & 73.1 \\
5 & 62.3 & 1.6 & 0.0 & 36.1 \\
6 & 59.4 & 1.5 & 0.0 & 39.1 \\
Rango & $96.5-98.5$ & $1.5-3.5$ & Hasta 1.0 & 0 \\
normal & & & & \\
\hline
\end{tabular}

* Valores por electroforesis.

tida y debutan con neumonía aun siendo leve, pueden presentar mal pronóstico si desconocen que presentan drepanocitosis en su variedad heterocigota u homocigota, aunado a ello un descontrol metabólico por la diabetes o hipertensión.

La inducción a drepanocitos se debe incluir como tamizaje de rutina a todos los pacientes con o sin anemia, especialmente aquéllos que presentan enfermedades respiratorias; cualquier persona es portadora de la enfermedad hasta que se demuestre lo contrario. Se debería informar a los portadores y a las parejas en riesgo de ese peligro y de las opciones para mitigarlo, pues cuando dos personas (hombre y mujer heterocigotas) que desconocen que presentan esta enfermedad y son asintomáticos, contraen matrimonio y agravan la enfermedad en sus descendientes. La finalidad es diagnosticar las formas no graves (heterocigotos), para así evitar las formas graves (homocigotos).

Finalmente, el diagnóstico de la anemia de células falciformes o anemia drepanocítica (hemoglobinopatía S), puede llegar a ser muy sencillo y oportuno, si los laboratorios clínicos implementan al menos la inducción a drepanocitos y la lectura del frotis sanguíneo, independientemente si la BHC es normal. El cribado de las hemoglobinopatías debería formar parte de los servicios básicos de salud en la mayoría de los países.

\section{AGRADECIMIENTOS}

Agradecemos enormemente a las becarias Xochil Velázquez-Gutiérrez y Karla Briseth Sánchez-Domínguez, así como las administrativa Ana María Velázquez-Gómez, por su valioso apoyo en la atención de los pacientes y el control de registros, que sin duda fueron de gran importancia para que cada dato e información estuviera al alcance y este trabajo se haya realizado sin demora.

\section{REFERENCIAS}

1. Paho.org. [Online] 2020; [Accessed 9 September 2020] Available at: https://www.paho.org/hq/index.php?option =com docman\&view=download\&category_slug=health-systems-servicessistemas-servicios-salud-5372\&alias $=21393$-estandares-trabajoservicios-sangre-tercera-edicion-393\&ltemid $=270 \&$ lang $=\mathrm{es}$.

2. Preguntas y respuestas sobre la enfermedad por coronavirus (COVID-19). [Recuperado 11 Septiembre 2020] Disponibleen: https:// www.who.int/es/emergencies/diseases/novel-coronavirus-2019/ advice-for-public/q-a-coronaviruses\#: :text=sintomas, 2020.

3. Herrick JB. Peculiar elongated and sickle-shaped red blood corpuscles in a case of severe anemia. Arch Inter Med. 2010; 6: 517.

4. Galiza G, Da Silva M. Aspectos moleculares da anemia falciforme. Bras Patol Med Lab. 2003; 39: 51-66.

5. Argüelles R. Fundamentos de Hematología. 4a ed. Buenos Aires, Argentina: Médica Panamericana, 2009.

6. Piel FB, Steinberg MH, Rees DC. Sickle Cell Disease. N Engl J Med 2017; 376:1561-73.

7. Chakravorty S, Williams TN. Sickle cell disease: a neglected chronic disease of increasing global health importance. Arch Dis Child 2015; 100:48-53.

8. National Institutes of Health. National Heart, Lung, and Blood Institute Division of Blood Diseases and Resources. The management of sickle cell disease. Fourth Edition. Bethesda, MD: NIH Publication 2015.

9. Mesa-Cuervo JR, Cháguez-Leyva O, Hechavarría-Miyares J, Plasencia-Ternblóm A, Losada-Buchillón R, Rodríguez LR et al. Modificaciones funcionales ventilatorias en pacientes con anemia drepanocítica y antecedentes de síndrome torácico agudo. Rev Cubana Hematol Inmunol Hemoter. 2016; 18 (2).

10. Fonseca CSV, Araújo-Melo CA, Matias de Carvalho R, Barreto-Neto J, Gonçalves Araújo J, Cipolotti R. Função pulmonar em portadores de anemia falciforme. Rev Paul Pediatr. 2017; 29 (1): 85-90.

11. Drepanocitosis | Federación Mexicana de Enfermedades Raras. [Recuperado 11 September 2020] Disponible en: http://www. femexer.org/2613/drepanocitosis/2020.

12. World Medical Association. Declaration of Helsinki: ethical principles for medical research involving human subjects. Edinburgh, 2015.

13. Secretaría de Salud. Ley General de Salud de México y disposiciones complementarias. Ciudad de México, México: Editorial Porrúa 1994.

14. OMS. Epidemiología mundial de las hemoglobinopatías e indicadores de los servicios correspondientes. [Recuperado 11 Septiembre 2020] Disponible en: https://www.who.int/bulletin/ volumes/86/6/06-036673-ab/es/.

15. Peñaloza-Espinosa, Rosenda I, Buentello-Malo, Leonor, HernándezMaya, Arcelia, Nieva-García, Beatriz, Lisker-Yurkowitzki, Rubén, \& Salamanca-Gómez, Fabio. Frecuencia de la hemoglobina S en cinco poblaciones mexicanas y su importancia en la salud pública. Salud Pública de México. 2016; 50 (4): 325-329. Recuperado en: 11 de septiembre de 2020, Disponible en: http:// www.scielo.org. $\mathrm{mx} /$ scielo.php?script=sci_arttext\&pid =S003636342008000400011\&lng=es\&tlng=es.

16. Hernández-González E. Hematología elemental "Lo que usted necesita saber" (anemia drepanocítica). 2ª ed. Veracruz, México. 2019, pp. 70-79. 